\title{
Multimedia information system on assistive devices
}

\author{
R.Andrich \\ SIVA, Fondazione Pro Juventute Don C.Gnocchi IRCCS \\ Milano, Italy
}

\begin{abstract}
A computerised information system with multimedia capabilities is described which supports a network of technical aids information and advice Centres in Italy. The system is currently used by 58 Centres disseminated throughout the Country, mainly run by Local Health Authorities of the National Health Service, and by 10 Centres abroad. The distribution medium is the compact disk CD-ROM. A number of other services (monthly telefaxed bulletins, training programmes, help desk) are provided along with the quarterly distribution of CD-ROMs.
\end{abstract}

\section{Background}

Information on the available assistive devices and related aspects (manufacturing, distribution, legislation etc...) plays a substantial role in the whole process of delivering appropriate technology for rehabilitation and independent living. For such purpose a number of specialised computerised information systems have been developed in many Countries, like most European Countries, USA, Canada, New Zealand, and some of them have a long lasting tradition.

SIVA launched in 1981 its own information system, which has been in use since then within SIVA's technical aids information and advice Centre.

Over the years a continuous improvement of the information system software and contents was carried out by SIVA's multidisciplinary team, on the ground of the advances in informatics and of the direct experience of providing information and advice to a wide variety of clients.

In 1988 decision was taken to move the system from a centralised approach (mainframe DEC VAX 11/750, also made available for remote access through modem and dial-up telephonic line) to a decentralised approach based on Personal Computer, responding to a highly increasing demand by Local Health Authorities (in Italian "USL"), Regional Governments, Associations of disabled persons involved in community services.

In 1992 the system entered its multimedia stage, by starting to manage pictorial information (pictures of technical aids) complementing textual information. 


\section{Design criteria}

On the ground of the previous experience and after getting deeper insight into the information needs of rehabilitation professionals, the data bank management system was completely rewritten for implementation onto the most widespread Personal Computers.

Advantage was taken by the most recent available techniques and programming languages. The most remarkable improvement consisted in associating pictorial information on products (technical aids) to factual data.

The possibility to get on the screen the picture of a given technical aid in addition to textual information had always been deemed of immense value when choosing a technical aid for meeting a need of a disabled individual. Until recently this possibility was hampered by the cost of the needed equipment in terms of either hardware or distribution media. Now the economic threshold has been overcome and an effective solution has been implemented.

That brought about the need for adopting a new distribution medium with suitable data storage capabilities (the compact disk CD-ROM), thus making the information system a powerful tool for assisting the rehabilitation professional in the choice of the most appropriate technical aid.

\section{Description of the system}

The current version of the computerised information requires a DOS (rel.6) compatible Personal Computer based on at least a 386 processor, equipped with:

- 4 Mbytes RAM memory

- $\quad$ SVGA colour screen able to display 256 colours

- $\quad 550$ Mbytes CD-ROM drive

- at least 25 Mbytes available in the hard disk.

The files format complies with DBIII format and the software management system was written by SIVA in Clipper language (release 5.1) for running directly in MS/DOS environment. The software interface is highly user friendly, its use does not require any informatics skill, its concept is especially addressed towards rehabilitation professionals.

The following retrieval functions are offered by the main menu:

\section{NATIONAL FILE (read only)}

A technical aids

B manufacturers, suppliers, retailers of technical aids

C associations, research centres, relevant institutions

$\mathrm{G}$ guide to technical aids classification

L legislation concerning disability 
$\mathrm{P} \quad$ arrangements for funding technical aids or house adaptations

$\mathrm{N}$ the Ministry Official List of assistive devices entitled to free-of-charge provision

B literature concerning tech.aids and accessibility

$\begin{array}{ll} & \text { LOCAL FILE (read/write) } \\ \mathrm{I} & \text { saving clients' records } \\ \mathrm{R} & \text { retrieving client's records } \\ \mathrm{S} & \text { statistics concerning clients' profile }\end{array}$

The data bank contains about 19000 records in the whole: almost 6000 of them describe products (technical aids), about 7000 describe commercial or non commercial organisation, the remaining ones refer to legislation, literature, explanations and miscellane-

Pict. 1 - SIVA CD-ROM ous. The data bank also includes:

- a guide to the selection of technical aids, based upon the ISO 9999 International Classification of Technical Aids, which allows the user to navigate through terminology and get suggestions on points to consider when choosing a technical aid. - the official national register of technical aids, listing those devices and orthopaedic appliances which can be provided by free of charge by the USL

- a special utility for keeping record of the counselling services given to the clients, based upon standardised forms which can be easily filled in. This facility includes also the possibility to record a follow up form which is generally sent to the client after some six months. Processing such data provides also statistics of the users profile, their impairments and pathologies, raised questions and proposed solutions. - a on-line user manual, accessible whenever pressing key F1.

Searching on technical aids (function " $A$ "), for instance, is possible by specifying up to 14 retrieval parameters (e.g. model, classification, thesaurus, sizes, functional limitation etc...), raising to 23 when searching on complex products like for instance wheelchairs (e.g. seat size etc...). At the end of a search the description of the retrieved products can be displayed on the screen or printed according to various formats. A typical product description shows up to 50 parameters including identification data, factual data, freetext, and a colour picture.

\section{Updating, management and distribution}

The production, the updating and the distribution of the data bank is carried out by a team which includes 2 experts in technical aids, 3 documentalists, 1 technician, 1 computer analyst/programmer, plus co-ordination and clerical support. In the whole the dedicated manpower is the equivalent of 6 full-time workers. 
Pictorial information concerning each product is taken from pictures provided by manufacturers (or from commercial catalogues), through a colour scanner or videocamera (Screen Machine rel.2) and commercial scanning or video-grabbing software. A minimal amount of graphic manipulation is then performed in order to comply with a set of consistency rules so as to ensure that the resulting image be understandable and informative for a rehabilitation professional. Each picture constitutes a file record which is stored onto a large-size-memory hard disk in FLM format (each file contains the pictures of all products belonging to a common brand name), from which it is converted into a PCX file with relevant links with the associated textual records everytime a new CD-ROM is prepared.

The system is designed for quarterly distribution through compact disk CD-ROM whose mastering is performed by a service agency. In order to have the whole information system implemented onto a single compact disk and fully usable with widespreads VGA colour screens, decision was taken:

- to limit the palette to 256 colours

- to have just one picture per product

- to have the image as large as possible on the screen so as to improve intelligibility and appreciation of details. Such compromise has been experienced as excellent between image quality, speed of screen presentation (almost instantaneous), required mass-memory (100-150 Kbytes for each picture).

Now compression techniques are being experienced in order to be able to manage more than one picture per product.

In retrieval stage access to the picture is provided by pressing an appropriate function key after textual information relevant to the given product is displayed.

\section{The information/advice centres network throughout Italy}

Assisting the client in the appropriate choice of a technical aid is a highly professional task. Therefore SIVA's Data Bank is mainly addressed at rehabilitation services and resource Centres, where experts are expected to be prepared to act as counsellors to disabled clients. Up to now 58 centres (scattered throughout 12 out of the 20 Italian Regions) are accessing the system and thus take part in this network. However their number is rapidly increasing. Most of them have been set up inside Rehabilitation Units of Local Health Authorities: the remainder are information and Resource Centres set up by Municipalities, Associations ad Universities. 14 of them are concentrated in the Region of Lombardy and 10 in the Region of Campania, in the frame of special projects aimed at providing every

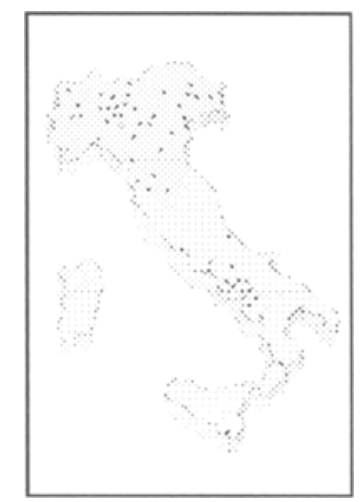

Pict.2 - SIVA netwodk 
Local Health Authority with a specialised information office able to deal with all matters concerning technical aids.

Subject to an annual subscription, Siva provides the data bank and updates it every fourth month by mailing of a new compact disc. Every update is a new version of the whole package (data and software) which supersedes the old one.

The subscription also includes a monthly bulletin which reports on relevant initiatives (congresses, courses, important news) in the field of disability and rehabilitation. In order to responding to the need for fresh and quick information on such events the bulletin is telefaxed: a cost-effective solution for that was found through an external agency which provides a computer-assisted fax dispatching system.

In addition to that, SIVA offers specific training programmes addressed at professionals of the local information Centres, concerned with a global approach to counselling on the choice of technical aids with the support of the data bank. A permanent help desk is ensured on the phone which assists the data bank users in installation problems and in conceptual data retrieval difficulties. One-and-half day training sessions are also offered to help the data bank users in improving their skills in searching on the system.

\section{Links with the European HANDYNET System}

SIVA has been involved in the development of the European information system HANDYNET since its inception in 1986, together with a number of research and rehabilitation Institutions in other European Countries. Now it is appointed as the national responsible for Handynet data collection and distribution in Italy. Through a purpose-made software interface data are transferred from SIVA information system whenever requested by the European Commission and reformatted so as to comply with the Handynet standards. The Handynet CD-ROMs and the related multilingual retrieval software are then distributed to all the Information Centres belonging to SIVA network, thus providing a powerful possibility to search for technical aids available on all the Common market.

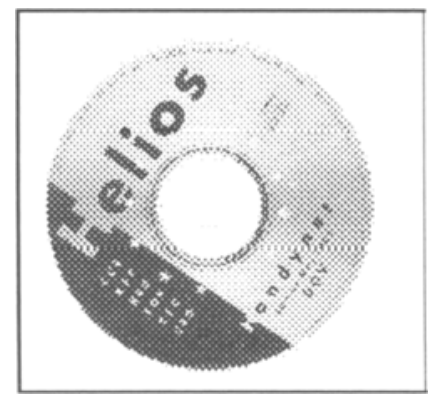

Pict. 3 - The Handynet CD-ROM

\section{Discussion}

The current version of the information system benefits of 13 years of experience in idevelopment, management, distribution and direct use as a tool for supporting information and advice service delivery on technical aids. International discussion, 
exchanges and research were also carried out concerning this topic within the framework of the EEC Handynet project.

Whenever an improvement was carried out concerning the data bank structure or software, a field test was performed by direct observation and collection of opinions among the data bank users, who now range to about 240 professionals. The information system has proven to be a substantial tool for rehabilitation professionals and technicaids advisers; moreover, the possibility to access pictures is felt by them as a decisive step forwards.

But it has also been confirmed that the potentialities of the Data Bank can be exploited fully only in the hands of persons showing a solid background in the field of technical aids. That encouraged SIVA to design and experience training programmes aimed at reinforcing such knowledge and introducing well-defined methodologies of Technical Aids Counselling assisted by an information system.

A 100 hours training curriculum is now provided to professionals of the Centres who wish to take part in the SIVA cooperation network of Technical Aids Centres. That ensures a common commitment to quality of information service delivery and suggests a possible model applicable also in other Countries.

\section{References}

Andrich R.: The provision of technical aids in Italy: present situation and new models. In: Lorentsen $O$. (ed.): Rehabilitation Engineering. Proceedings of the UN/ECE REHAB-2 Workshop (Fagernes 12-15/5/91). Amsterdam: IFMBE 1992, pp. 73-87

Andrich R.: Educational programme for professionals and information providers. In: Technology and Accessibility. Proceedings of the ICTA international conference (Hoensbroek 12-13/11/91). Hoensbroek: Lucas Stichting 1992, pp.70-75 